\title{
ON DERIVATIONS OF CERTAIN ALGEBRAS RELATED TO IRREDUCIBLE TRIANGULAR ALGEBRAS
}

BY

\author{
BARUCH SOLEL
}

\begin{abstract}
This paper deals with derivations on algebras that are generated by a maximal abelian selfadjoint algebra of operators $\mathcal{Q}$ on a Hilbert space and a group of unitary operators acting on it. A necessary and sufficient condition for such a derivation to be implemented by an operator affiliated with $Q$ is given. The results are related to the study of derivations on a certain class of irreducible triangular algebras.
\end{abstract}

1. Introduction. This paper continues the study of derivations on a certain class of algebras of operators on a Hilbert space that started in [8]. In [8] we studied the structure of a class of irreducible triangular algebras and the $C^{*}$-algebras generated by those algebras. The irreducible triangular algebras are those generated by a maximal abelian algebra $\mathcal{Q}$ and an ordered semigroup $G$ of unitary operators acting on $Q$.

The investigation in [8] follows two paths. Along the first, it is a further development of the structure theory of a subclass of nonselfadjoint operator algebras - the irreducible triangular algebras. Along the second, it is an exploration of some parts of noncommutive ergodic theory - with emphasis on nonselfadjoint features of the theory.

The study of triangular operator algebras was initiated by Kadison and Singer in a paper [4] which appeared in 1960. With $H$ a complex Hilbert space and $B(H)$ the algebra of all bounded operators on it, a subalgebra $\delta$ of $B(H)$ such that $\delta \cap \delta^{*}$ is maximal abelian in $B(H)$ is said to be triangular and $\delta \cap \delta^{*}$ is said to be its diagonal.

If the only projections $E$ in $B(H)$ that are left invariant by each operator $T$ in $\mathcal{S}$ (i.e. $E T E=T E$ ) are $E=0$ and $E=I$, then the algebra $\delta$ is said to be irreducible.

As proved in [8, Corollary 1.5], if $G$ is an ordered semigroup of unitary operators acting freely and ergodically on a maximal abelian algebra $\mathcal{Q}$, then the algebra $\mathcal{S}$, generated by $Q$ and $G$, is an irreducible triangular algebra.

The derivations and automorphisms of $\delta$ are closely related to the skewadjoint derivations and the ${ }^{*}$-automorphisms on the *-algebra $\delta+\mathcal{S}^{*}$. Those objects are studied in Chapter IV of [8] (under the further assumption that the *automorphisms leave each operator in $\mathscr{Q}$ fixed, and the derivations vanish on $\mathscr{Q}$ ). The group of *automorphisms of $\mathcal{S}+S^{*}$ that leave each operator in $\mathbb{Q}$ fixed will be denoted

Received by the editors December 1, 1981 and, in revised form, April 22, 1982.

1980 Mathematics Subject Classification. Primary 46L05; Secondary 47D25, 46K05, 46L99. 
$\operatorname{Aut}(\mathscr{B}, \mathscr{Q})\left(\mathscr{B}\right.$ is the $C^{*}$-algebra generated by $\mathcal{S}$ ). The set of all skewadjoint derivations on $\delta+\delta^{*}$ that vanish on $\mathbb{Q}$ will be denoted $D\left(\delta+\delta^{*}, \mathbb{Q}\right)$. A map $\varepsilon$ is defined, from $D\left(\delta+\mathcal{S}^{*}, \mathscr{Q}\right)$ into $\operatorname{Aut}(\mathscr{B}, \mathcal{Q})$, such that $\varepsilon(\delta)(T)=(\exp (i \delta))(T)$ for each $T$ in $\delta+\delta^{*}$.

For the next result we will assume that the group generated by $G$ is amenable.

For a derivation $\delta$ in $D\left(\delta+\mathcal{S}^{*}, \mathscr{Q}\right)$ we proved the equivalence of the following conditions (see [8, Lemma 4.12 and Theorem 4.20]):

(1) $\delta$ is bounded.

(2) There is an operator $D$ in $\mathcal{Q}$ implementing $\delta$ (i.e. $\delta(T)=D T-T D, T \in \mathcal{S}+$ S*).

(3) $\operatorname{Sup}\{\|\delta(U)\|: U \in G\}<\infty$.

We present here a different proof of this fact (Theorem 2.2) using averaging techniques (see [6, Lemma 4.2]).

The main result of this paper is Theorem 4.7 which gives a necessary and sufficient condition for a derivation in $D\left(\mathcal{S}+\delta^{*}, \mathcal{Q}\right)$ to be "implemented" by a linear, selfadjoint (not necessarily bounded) operator affiliated with $\mathscr{Q}$.

For this, we will analyze groups of automorphisms on the algebra $P \Re P$, for $P$ a projection in $Q$. This is done in $\$ 3$.

2. Preliminaries. We now describe the notions and the results basic to the remaining work.

We will deal with the action of a semigroup of unitary operators on a maximal abelian von Neumann algebra. For this, we define an ordered (unitary) semigroup to be a semigroup $G$ such that:

(1) $G \cup G^{-1}$ is a group, to be denoted by $\bar{G}$.

(2) $G \cap G^{-1}=\{I\}$ where $I$ is the unit element.

(3) For each $W$ in $\bar{G}, W G W^{-1}=G$.

Henceforth $X$ will denote a locally compact Hausdorff space and $m$ a $\sigma$-finite regular Borel measure on $X$. Let $H$ be the Hilbert space $L^{2}(X, m)$ and $B(H)$ be the algebra of all bounded linear operators acting on $H$. For each function $f$ in $L^{\infty}(X, m)$ define the operator $L_{f}$ in $B(H)$ by $L_{f} g=f g$ (multiplication by $f$ ). The algebra $Q=\left\{L_{f}: f \in L^{\infty}(X, m)\right\}$ is a maximal abelian subalgebra of $B(H)$. Every unitary operator $U$ that satisfies $U^{*} \mathbb{Q} U=\mathbb{Q}$ is said to act on $\mathbb{Q}$, the action being $A \rightarrow U^{*} A U$.

We say that $U$ acts freely on $Q$ if for each nonzero projection $Q$ in $Q$, there is a nonzero projection $E$ in $Q$ such that $E \leqslant Q$ and $E U^{*} E U=0$. We say that a semigroup $G$ acts freely on $Q$ when each $U$ in $G$, other than $I$, acts freely on $Q$.

From now on, $G$ will be an ordered semigroup of unitary operators in $B(H)$ and $\mathcal{S}$ will be the algebra (not necessarily closed or selfadjoint) generated by $\mathcal{Q}$ and $G$. The *-algebra generated by $\mathcal{Q}$ and $\bar{G}$ is $\mathcal{S}+\mathcal{S}^{*}$.

Definitions. (1) We say that $G$ acts ergodically on $Q$ (or that $G$ is ergodic) if for each nonzero projection $P$ in $Q, I=\vee\left\{U^{*} P U: U \in G\right\}$.

(2) An algebra $\mathcal{S}$ of operators on a Hilbert space $H$ is called irreducible if Lat $\delta=\{0, I\}$ where Lat $\delta=\{P \in B(H): P$ is a projection and $P T P=T P, T \in$ S\}. 
THEOREM 2.1 [8, COROLlARY 1.5]. Let $G$ be an ordered semigroup acting freely and ergodically on $\mathcal{Q}$. Then $\mathcal{S}$ is an irreducible triangular algebra.

The main step in the proof of the theorem is the "Q property that if $A_{i}$ are in $Q$ and $U_{i}$ are in $\bar{G}$, then

$$
\sum_{i=1}^{n} A_{i} U_{i}=0 \text { implies } A_{i}=0, \quad 1 \leqslant i \leqslant n .
$$

We will assume, throughout this paper, that $G$ is an ordered semigroup of unitary operators acting freely and ergodically on $\mathcal{Q}$. Furthermore, we assume that $\bar{G}$ is an amenable group (i.e. there is a finitely additive probability measure $\mu$ on the field of all subsets of $\bar{G}$ such that $\mu(x E)=\mu(E)$ for all $x \in \bar{G}, E \subseteq \bar{G})$.

We now turn to study the derivations on $\mathcal{S}+\mathcal{S}^{*}$. A skewadjoint derivation $\delta$ on a *-algebra $\Re$ is a linear map, from $\Re$ into itself, satisfying:

(1) $\delta(a b)=\delta(a) b+a \delta(b), a, b \in \mathfrak{N}$.

(2) $\delta\left(a^{*}\right)=-(\delta(a))^{*}, a \in \mathfrak{N}$.

We let $D\left(\delta+\delta^{*}\right)$ denote the set of all skewadjoint derivations on $\mathcal{S}+\delta^{*}$.

THEOREM 2.2. Let $\delta$ be a derivation in $D\left(\delta+\delta^{*}\right)$ such that:

(1) Its restriction to $Q$ is bounded.

(2) $\operatorname{Sup}\{\|\delta(U)\|: U \in \bar{G}\}<\infty$.

Then there is an operator $S$ in $B(H)$ such that

$$
\delta(T)=S T-T S, \quad T \in \mathcal{S}+\mathcal{S}^{*} .
$$

Proof. Let $\mathscr{Q}$ be the unitary group of $\mathcal{Q}$, and let $\mathfrak{V}$ be the group generated by $\mathscr{U}$ and $\bar{G}$. Since each $U$ in $\bar{G}$ acts on $\mathcal{Q}, \mathcal{Q}$ is a normal subgroup of $\mathscr{V}$ and $\mathscr{V} / \mathcal{Q}$ is isomorphic to $\bar{G}$ via the map $V U \mathcal{Q} \rightarrow U, V \in \mathcal{Q}, U \in \bar{G}$. (We use here the Q-independence of $\bar{G}$, mentioned above.) Since both $\bar{G}$ and $\mathcal{U}$ are amendable $(\bar{G}$ is amenable by assumption and $\mathscr{U}$ is commutative and, hence, amenable by $[3$, Theorem 1.2.1]) $\mathcal{T}$ is also amenable (see [3, Theorem 1.2.6]).

Let $\mathrm{BC}(\mathcal{V})$ be the Banach space of all bounded continuous functions from $\mathfrak{V}$ into $B(H)$, with the norm

$$
\|t\|=\operatorname{Sup}\{\|t(W)\|: W \in \mathfrak{V}\} .
$$

By [6, Lemma 4.2] there is a norm decreasing function $g$ from $\mathrm{BC}(\mathfrak{V})$ into $B(H)$, such that:

(i) If $V, U \in \mathcal{V}, t \in \mathrm{BC}(\mathcal{V})$ and $t^{\prime}(W)=V t(W) U$ for all $W$ in $\mathcal{T}$, then $g\left(t^{\prime}\right)=V g(t) U$.

(ii) If $V \in \mathscr{V}, t \in \mathrm{BC}(\mathcal{V})$ and $t_{V}(W)=t(V W)$ for all $W$ in $\mathcal{V}$, then $g\left(t_{V}\right)=g(t)$.

(iii) $g(t)=R$ if $t(W)=R$ for all $W$ in $\mathscr{T}$.

We will use this result for $t \in \mathrm{BC}(\mathfrak{V})$ defined by

$$
t(W)=\delta(W) W^{*} .
$$

To show that $t$ is in $\mathrm{BC}(\mathfrak{V})$, note first that $t$ is bounded (by the hypothesis of the theorem). It is also a continuous map. To see this, let $V_{n} U_{n} \rightarrow V U, V_{n}, V \in \mathcal{Q}, U_{n}$, $U \in \bar{G}$. By [8, Lemma 2.15], if $\left\|V_{n} U_{n}-V U\right\|<\sqrt{2}$, then $U_{n}=U$. So we can assume 
that $U_{n}=U$ for all $n$ and $V_{n} \rightarrow V$, in $Q$. But $\delta$ is continuous on $Q$, hence

$$
\begin{aligned}
t\left(V_{n} U_{n}\right) & =t\left(V_{n} U\right)=\delta\left(V_{n} U\right) U^{*} V_{n}^{*} \\
& =\delta\left(V_{n}\right) V_{n}^{*}+V_{n} \delta(U) U^{*} V_{n}^{*} \rightarrow \delta(V) V^{*}+V \delta(U) U^{*} V^{*}=t(V U) .
\end{aligned}
$$

Therefore we can let $S$ be $g(t)$. Then for all $V, W \in \mathscr{V}, t_{V}(W)=t(V W)=$ $\delta(V U) U^{*} V^{*}=\delta(V) V^{*}+V \delta(W) W^{*} V^{*}$ and $S=g(t)=g\left(t_{V}\right)=\delta(V) V^{*}+V S V^{*}$. Thus

$$
\delta(V)=S V-V S, \quad V \in \mathcal{T} .
$$

Since $\mathscr{T}$ spans $\mathcal{S}+\mathfrak{S}^{*}$, as a linear space,

$$
\delta(T)=S T-T S, \quad T \in \mathcal{S}+\mathcal{S}^{*} .
$$

In Theorem 4.7 we will generalize this result (with the assumption that $\delta \mid \mathbb{Q}=0$ ) by imposing a weaker condition $\operatorname{than} \operatorname{Sup}\{\|\delta(U)\|: U \in \bar{G}\}<\infty$. As a result, the operator $S$ will be replaced by an unbounded operator.

3. Automorphisms of the algebra $P \mathscr{B}$. Let $P$ be a nonzero projection in $\mathcal{Q}$. By [8, Proposition 2.22] the algebra $P S P$ is an irreducible triangular algebra. Let $P\left(\mathcal{S}+\delta^{*}\right) P$ denote the selfadjoint algebra generated by $P \delta P$, and $P \mathscr{B} P$ its norm closure $\left(P \mathscr{P} P\right.$ is a $C^{*}$-algebra).

Let $\operatorname{Aut}(P \Re P, P Q)$ denote the set of all the ${ }^{*}$-automorphisms on $P \Re P$ leaving each member of $P Q$ fixed.

LEMma 3.1. For $\psi \in \operatorname{Aut}(P \mathscr{B} P, P Q)$ there is a map $\varphi$ from $\bar{G}$ into $P \mathscr{Q}$ such that for each $U$ in $\bar{G}$,

$$
\psi(P U P)=\varphi(U) P U P .
$$

Proof. Fix $\psi$ in $\operatorname{Aut}(P \Re P, P Q)$ and $U$ in $\bar{G}$. For each $A$ in $Q$,

$$
\psi(P A U P)=\psi(P A) \psi(P U P)=P A \psi(P U P)
$$

and $\psi(P A U P)=\psi\left(P U P U^{*} A U P\right)=\psi(P U P) \psi\left(P U^{*} A U P\right)=\psi(P U P) P U^{*} A U P$. Hence

$$
P A \psi(P U P)=\psi(P U P) P U^{*} A U
$$

and

$$
A \psi(P U P) U^{*}=\psi(P U P) U^{*} A .
$$

Thus $\psi(P U P) U^{*} \in \mathbb{Q}^{\prime}=\mathbb{Q}$ and, since $\psi(P U P)=P \psi(P U P), \psi(P U P) U^{*}$ is in $\mathbb{Q} P$.

We can write $\psi(P U P)=B U$ for some $B$ in $Q P$, and we have $P A B U=B U P U^{*} A U$ (by (*)) for each $A$ in $Q$. In particular $B U=P B U=B U P$ (let $A=I$ ) and $B=$ $B U P U^{*}$. Hence $B \in \mathbb{Q} P U P U^{*}$ and we can write $B=\varphi(U) P U P U^{*}$. This implies that $\psi(P U P)=\varphi(U) P U P$.

Let $\alpha: t \rightarrow \alpha_{t}$ be a homomorphism from $\mathbf{R}$ into $\operatorname{Aut}(P \mathscr{B} P, P Q)$ and assume that $\alpha_{t}$ is implemented by $\varphi_{t}$ (in the sense of the previous lemma). Assume also that for each $T$ in $P \Re P$, the map $t \rightarrow \alpha_{t}(T)$ is norm-continuous.

In particular, $t \rightarrow \varphi_{t}(U) P U P U^{*}$ is norm-continuous. Let $\varphi_{t}^{\prime}(U)$ be $P-P U P U^{*}+$ $\varphi_{t}(U) P U P U^{*}$, then $\varphi_{t}^{\prime}(U)$ is a unitary operator in $P Q$ (acting on $P(H)$ ), and the map $t \rightarrow \varphi_{t}^{\prime}(U)$ is a norm-continuous one-parameter unitary group. (Since $\varphi_{t}^{\prime}(U) \varphi_{s}^{\prime}(U)=$ $\left.P-P U P U^{*}+\varphi_{t}(U) \varphi_{s}(U) P U P U^{*}=P-P U P U^{*}+\varphi_{t+s}(U) P U P U^{*}=\varphi_{t+s}^{\prime}(U).\right)$ 
Hence there is a selfadjoint operator $C(U)$, in $P Q$, such that $\varphi_{t}^{\prime}(U)=\exp (i t C(U))$ (see [2, Theorem VIII.1.2]).

Let $M(\mathbf{R})$ denote the Banach space of all complex finite regular Borel measures on $\mathbf{R}$ with the total variation as the norm. For each $f$ in $L^{1}(\mathbf{R})$ the measure $f(t) d t$ is in $M(\mathbf{R})$ and its total variation equals $\|f\|_{1}$. For each $\mu$ in $M(\mathbf{R})$ and $T$ in $P \Re P$ there is a unique bounded operator $\alpha_{\mu}(T)$ in $\Re$ such that, for each $g$ in the dual space of $P \Re P$

$$
g\left(\alpha_{\mu}(T)\right)=\int_{\mathbf{R}} g\left(\alpha_{t}(T)\right) d \mu(t) .
$$

Also, for each $\mu$ in $M(\mathbf{R})$ and $U$ in $\bar{G}$, there is a unique bounded operator $\varphi_{\mu}^{\prime}(U)$ in $\mathbb{Q} P$ such that for each $g$ in the dual space of $\mathscr{P}$,

$$
g\left(\varphi_{\mu}^{\prime}(U)\right)=\int_{\mathbf{R}} g\left(\varphi_{t}^{\prime}(U)\right) d \mu(t) .
$$

We will denote $\varphi_{\mu}^{\prime}(U)$ as $\int_{\mathbf{R}} \varphi_{t}^{\prime}(U) d \mu(t)$. For details on the last two statements see [1, Proposition 1.2 or $\mathbf{8}$, Corollary 4.2].

When the measure $\mu$, in $M(\mathbf{R})$, is the measure $f(t) d t$ (for some $f \in L^{1}(\mathbf{R})$ ) we use the notations $\varphi_{f}^{\prime}$ and $\alpha_{f}$ for $\varphi_{\mu}^{\prime}$ and $\alpha_{\mu}$ respectively.

We will employ analysis, similar to the analysis in $[8$, Chapter IV], for the algebra $P \Re P$.

Lemma 3.2. For every $\mu$ in $M(\mathbf{R}), U$ in $\bar{G}$, and $A$ in $P Q, \alpha_{\mu}(A P U P)=A \varphi_{\mu}^{\prime}(U) P U P$.

Proof. Fix $U$ in $\bar{G}, A$ in $P Q$, and $\mu$ in $M(\mathbf{R})$. For $g$ in the dual of $P \Re P$,

$$
g\left(\alpha_{\mu}(A P U P)\right)=\int_{\mathbf{R}} g\left(\alpha_{t}(A P U P)\right) d \mu(t)=\int_{\mathbf{R}} g\left(A \varphi_{t}^{\prime}(U) P U P\right) d \mu(t) .
$$

Let $g_{0}$ be defined by $g_{0}(B)=g(B A P U P)$. As $g$ is linear, $g_{0}$ is linear and

$$
\left|g_{0}(B)\right|=|g(B A P U P)| \leqslant\|g\|\|B\|\|A\| ;
$$

so that $g_{0}$ is in the dual of $P Q$. By the definition of $\varphi_{\mu}^{\prime}$ we now have

$$
\begin{aligned}
g\left(\alpha_{\mu}(A P U P)\right) & =\int_{\mathbf{R}} g_{0}\left(\varphi_{t}^{\prime}(U)\right) d \mu(t) \\
& =g_{0}\left(\varphi_{\mu}^{\prime}(U)\right)=g\left(A \varphi_{\mu}^{\prime}(U) P U P\right) .
\end{aligned}
$$

Since this holds for each $g$ in the dual of $P \Re P$ we obtain $\alpha_{\mu}(A P U P)=A \varphi_{\mu}^{\prime}(U) P U P$.

Let $\Omega$ be an open set in $\mathbf{R}$. Denote by $K(\mathbf{R}, \Omega)$ the set of functions $f$ such that the support of $\hat{f}$ (the Fourier transform of $f$ ) is compact and contained in $\Omega$.

For a closed subset $Z$ of $\mathbf{R}$ we define $M^{\alpha}(Z)$ to be the set of all operators $T$ in $P \Re P$ such that $\alpha_{f}(T)=0$ for each $f$ in $K(\mathbf{R}, \mathbf{R} \backslash Z)$.

LEMMA 3.3. $M^{\alpha}(Z)=P \Re P$ if and only if for each $f$ in $K(\mathbf{R}, \mathbf{R} \backslash Z)$ and each $U$ in $\bar{G}, P U P U^{*} \varphi_{f}^{\prime}(U)=0$.

Proof. Assume, first, that $M^{\alpha}(Z)=P \mathscr{P}$. Then, for each $f$ in $K(\mathbf{R}, \mathbf{R} \backslash Z)$ and each $U$ in $\bar{G}, \alpha_{f}(P U P)=0$. Hence $0=\alpha_{f}(P U P)=\varphi_{f}^{\prime}(U) P U P$. 
For the other direction, note that $M^{\alpha}(Z)$ is closed in the norm topology hence it will suffice to show that $P\left(\delta+\delta^{*}\right) P$ is contained in $M^{\alpha}(Z)$.

But every $T$ in $P\left(\delta+\varsigma^{*}\right) P$ has the form $\Sigma A_{U} P U P$ (where $A_{U}$ are in $\mathcal{Q}$ ) and, therefore, for each $f$ in $K(\mathbf{R}, \mathbf{R} \backslash Z), \alpha_{f}(T)=\Sigma A_{U} \varphi_{f}^{\prime}(U) P U P=0$. Thus $T$ is in $M^{\alpha}(Z)$.

The spectrum of $\alpha$ is defined as the smallest closed set $Z$ in $\mathbf{R}$ such that $M^{\alpha}(Z)=P \Re P$ and is denoted by $\operatorname{sp}(\alpha)$. For the spectrum of an operator $T$ we will use the notation $\sigma(T)$.

THEOREM 3.4. Let $t \rightarrow \alpha_{t}$ be a homomorphism from $\mathbf{R}$ into $\operatorname{Aut}(P \Re P, P Q)$ such that $\alpha_{t}$ is implemented by $\varphi_{t}^{\prime}$ and for each operator $T$ in $P \Re P$ the map $t \rightarrow \alpha_{t}(T)$ is norm-continuous. For $U$ in $\bar{G}$, let $C(U)$ be the operator in $P Q$ such that $\exp ($ it $C(U))=$ $\varphi_{t}^{\prime}(U)$, then

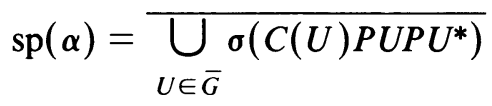

where $\bar{Y}$ denotes the closure of the set $Y$.

Proof. (a) Fix $U$ in $\bar{G}$. If $t^{\prime}$ is in $\sigma\left(C(U) P U P U^{*}\right)$ but not in $\operatorname{sp}(\alpha)$ then there is an $f$ in $K(\mathbf{R}, \mathbf{R} \backslash \operatorname{sp}(\alpha))$ with $\hat{f}\left(t^{\prime}\right)=1$. Since $M^{\alpha}(\operatorname{sp}(\alpha))=P \Re P$, Lemma 3.3 implies that $\varphi_{f}^{\prime}(U) P U P U^{*}=0$; hence

$$
\begin{aligned}
0 & =\varphi_{f}^{\prime}(U) P U P U^{*}=\int_{\mathbf{R}} \varphi_{t}^{\prime}(U) P U P U^{*} f(t) d m(t) \\
& =\int_{\mathbf{R}} \exp \left(i t C(U) P U P U^{*}\right) f(t) d m(t) .
\end{aligned}
$$

(Note that $f \in K\left(\mathbf{R}, \mathbf{R} \backslash \operatorname{sp}(\alpha)\right.$ ) implies $\int_{\mathbf{R}} f(t) d m(t)=0$.) Since $t^{\prime}$ is in $\sigma\left(C(U) P U P U^{*}\right)$, there is a pure state $\tau$ of $P Q$ such that $t^{\prime}=\tau\left(C(U) P U P U^{*}\right)$. Then

$$
\begin{aligned}
0 & =\tau\left(\varphi_{f}^{\prime}(U) P U P U^{*}\right)=\int_{\mathbf{R}} \exp \left(i t \tau\left(C(U) P U P U^{*}\right)\right) f(t) d m(t) \\
& =\int_{\mathbf{R}} \exp \left(i t t^{\prime}\right) f(t) d m(t)=\hat{f}\left(t^{\prime}\right) .
\end{aligned}
$$

But this contradicts the choice of $t^{\prime}$ and so proves that $\sigma\left(C(U) P U P U^{*}\right)$ is contained in $\operatorname{sp}(\alpha)$. Since $U$ is arbitrary (in $\bar{G})$ and since $\operatorname{sp}(\alpha)$ is a closed set,

$$
\bigcup_{U \in \bar{G}} \sigma\left(C(U) P U P U^{\prime}\right) \subseteq \operatorname{sp}(\alpha) .
$$

(b) Let $Z$ denote $\overline{\bigcup_{U \in \bar{G}} \boldsymbol{\sigma}\left(C(U) P U P U^{*}\right)}$ and let $f$ be in $K(\mathbf{R}, \mathbf{R} \backslash Z)$. Fix $U$ in $\bar{G}$, then for each pure state $\tau$ of $P Q$, we have $\tau\left(\varphi_{f}^{\prime}(U) P U P U^{*}\right)=\hat{f}\left(\tau\left(C(U) P U P U^{*}\right)\right)=$ 0 , thus $\varphi_{f}^{\prime}(U) P U P U^{*}=0$. Since this holds for each $U$ in $\bar{G}$, it implies, by Lemma 3.3, that $M^{\alpha}(Z)=P \mathscr{B}$. Hence $\operatorname{sp}(\alpha) \subseteq Z$ and the proof is complete.

4. Unbounded derivations on $\delta+\delta^{*}$. A derivation $\delta$ on an algebra $\mathfrak{T}$ is a linear map from the algebra into itself satisfying: For each $a, b$ in $\mathfrak{N}$

$$
\delta(a b)=\delta(a) b+a \delta(b) .
$$


The derivation will be said to be skewadjoint if for any $a$ in the algebra $\mathfrak{K}$, $\delta\left(a^{*}\right)=-\delta(a)^{*}$.

We denote by $D\left(\mathcal{S}+\mathcal{S}^{*}\right)$ the set of the skewadjoint derivations on $\mathcal{S}+\mathcal{S}^{*}$ and by $D\left(\mathcal{S}+\delta^{*}, \mathbb{Q}\right)$ the set of the skewadjoint derivations on $\delta+\delta^{*}$ that vanish on $Q$.

For a nonzero projection $P$ in $\mathcal{Q}$ and a derivation $\delta$ in $D\left(\delta+\delta^{*}, \mathcal{Q}\right)$ we let $\delta_{P}$ be the restriction of $\delta$ to $P\left(\delta+\delta^{*}\right) P$. As $\delta_{P}(P T P)=\delta(P T P)=P \delta(T) P, \delta_{P}$ is a skewadjoint derivation on $P\left(\mathcal{S}+\mathcal{S}^{*}\right) P$.

By [8, Lemma 4.11], there is a map $C$ from $\bar{G}$ into the selfadjoint operators of $Q$ such that for each $A$ in $Q$ and each $U$ in $\bar{G}, \delta(A U)=A C(U) U$. Therefore for $A$ in $P Q$ and $U$ in $\bar{G}$,

$$
\delta(A P U P)=A C(U) P U P .
$$

Such a map $C$, associated with $\delta$, is said to implement $\delta$.

Proposition 4.1. If $\delta$, in $D\left(\mathcal{S}+\mathcal{S}^{*}, \mathcal{Q}\right)$, is implemented by $C$ and if

$$
\operatorname{Sup}\left\{\left\|C(U) P U P U^{*}\right\|: U \in \bar{G}\right\}<\infty
$$

then $\delta_{P}$ is a bounded derivation.

Proof. By [8, Lemma 4.14] there is a homomorphism $\alpha^{\prime}$ from $\mathbf{R}$ into $\operatorname{Aut}(\mathscr{B}, \mathbb{Q})$ such that for each $T$ in $\delta+\delta^{*}, \alpha_{t}^{\prime}(T)=\exp (i t \delta)(T)$.

Let $\alpha: \mathbf{R} \rightarrow \operatorname{Aut}(P \mathscr{B}, P Q)$ be defined by the restriction of $\alpha^{\prime}$ to $P \mathscr{B} P$. Since $\alpha_{t}^{\prime}(P T P)=P \alpha_{t}^{\prime}(T) P, \alpha$ is well defined. By [8],

$$
\alpha_{t}(P U P)=\alpha_{t}^{\prime}(P U P)=(\exp (i t C(U))) P U P .
$$

Let $\varphi_{t}(U)$ be $\exp \left(i t\left(C(U) P U P U^{*}\right)\right)$ then $\alpha_{t}(P U P)=\varphi_{t}(U) P U P$.

Thus we can apply Theorem 3.4 to get

$$
\operatorname{sp}(\alpha)=\bigcup_{U \in \bar{G}} \sigma\left(C(U) P U P U^{*}\right) .
$$

By the hypothesis of the proposition, $\operatorname{sp}(\alpha)$ is compact and hence, by [5, Theorem 8.1.12], the map $t \rightarrow \alpha_{t}$ is norm-continuous. Using [2, Theorem VIII.1.2] there is a bounded map $\eta$ on $P \mathscr{B} P$ satisfying $\alpha_{t}=\exp (i t \eta)$ and $\eta=\lim _{t \rightarrow 0} \frac{1}{t}\left(\alpha_{t}-\right.$ id) where id is the identity automorphism and the limit is in the norm topology.

For each $A$ in $P Q$ and $U$ in $\bar{G}$ we have

$$
\begin{aligned}
\eta(A P U P) & =\lim _{t \rightarrow 0} \frac{1}{t}\left(\alpha_{t}(A P U P)-A P U P\right) \\
& =\lim _{t \rightarrow 0} \frac{1}{t}(A \exp (i t C(U)) P U P-A P U P) \\
& =A C(U) P U P=\delta_{P}(A P U P) .
\end{aligned}
$$

By linearity $\eta=\delta_{P}$ on $P\left(\delta+\delta^{*}\right) P$ and, therefore, $\delta_{P}$ is bounded.

We say that $\delta$ has a bounding sequence if there is a sequence of projections $\left\{P_{n}\right\}$ in $\mathcal{Q}$ such that $P_{n} \uparrow I$ and, for every $n, \operatorname{Sup}\left\{\left\|\delta\left(P_{n} U P_{n}\right)\right\|: U \in \bar{G}\right\}<\infty$.

Assume now that $\delta$ has a bounding sequence $\left\{P_{n}\right\}$ such that $P_{1} U P_{1} U^{*} \neq 0$. Proposition 4.1 shows that $\delta_{P_{n}}$ is a bounded derivation on $P_{n}\left(\delta+\delta^{*}\right) P_{n}$. We can extend $\delta_{P_{n}}$ and view it as a derivation on the $C^{*}$-algebra $P_{n} \Re P_{n}$. Thus there is an 
operator $D_{n}$ (acting on $P_{n}(H)$ ) that is selfadjoint and satisfies $\delta_{P_{\mathrm{n}}}=\operatorname{ad}\left(D_{n}\right)$ (see [7, Corollary 4.1.7]). Since $\delta_{P_{n}}\left(P_{n} \mathbb{Q}\right)=0$ and $P_{n} \mathbb{Q}$ is a maximal abelian algebra on $P_{n}(H), D_{n} \in P_{n} \mathbb{Q}$.

LEMMA 4.2. For $m>n, \delta_{P_{n}}=\operatorname{ad}\left(D_{m} P_{n}\right)$ and there is $r \in \mathbf{R}$ such that $D_{m} P_{n}-D_{n}=$ $r P_{n}$.

Proof. For $T$ in $P_{n}\left(\delta+\delta^{*}\right) P_{n}$,

$$
\begin{aligned}
\delta_{P_{n}}(T) & =\delta(T)=\delta\left(P_{m} T P_{m}\right)=\delta_{P_{m}}(T)=D_{m} T-T D_{m} \\
& =D_{m} P_{n} T-T D_{m} P_{n}=\operatorname{ad}\left(D_{m} P_{n}\right)(T) .
\end{aligned}
$$

Since this shows that $\operatorname{ad}\left(D_{n}\right)=\operatorname{ad}\left(D_{m} P_{n}\right)$ we see that $D_{n}-D_{m} P_{n}$ commutes with $P_{n}\left(\mathcal{\delta}+\varsigma^{*}\right) P_{n}$. The irreducibility of $P_{n} \delta P_{n}$ completes the proof.

If $\left\{D_{n}^{\prime}\right\}$ is a sequence of selfadjoint operators satisfying:

$$
\delta_{P_{n}}=\operatorname{ad}\left(D_{n}^{\prime}\right) \text { on } P_{n}\left(\delta+\delta^{*}\right) P_{n},
$$

then there is a sequence $\left\{r_{n}\right\}$ of real numbers such that $D_{n}^{\prime} P_{1}-D_{1}^{\prime}=r_{n} P_{1}$. Let $D_{n}$ be $D_{n}^{\prime}-r_{n} P_{n}$, then $D_{n} P_{1}=D_{1}$ and, for $m>n$,

$$
D_{m} P_{n}=D_{n} \text {. }
$$

A closed, densely defined, linear operator $T$ is affiliated with a von Neumann algebra $\Re$ if $U^{*} T U=T$ for each unitary operator $U$ in the commutant of $\Re$.

THEOREM 4.3. Let $\delta$ be a derivation in $D\left(\mathcal{S}+\mathcal{S}^{*}, \mathcal{Q}\right)$ with a bounding sequence $\left\{P_{n}\right\}$. Then there is a selfadjoint linear operator $D$, affiliated with $\mathbb{Q}$, such that for every $U$ in $\bar{G}$ and $f$ in $\mathscr{D}(D) \cap U^{*} \mathscr{D}(D)$,

$$
\delta(U) f=D U f-U D f .
$$

If $U f$ is in $\mathscr{D}(D)$ for every $U$ in $\bar{G}$, then for each $T$ in $\mathcal{S}+\delta^{*}, \delta(T) f=D T f-T D f$.

Proof. Recall that $X$ is a locally compact Hausdorff space with a $\sigma$-finite regular Borel measure $m, H$ is $L^{2}(X, m)$ and $Q$ is the multiplication algebra on $H$.

For each $n, P_{n}$ is the operator of multiplication by the characteristic function of some measurable set $E_{n}$ of $X$. Since $P_{n} \uparrow I$, we can assume that $E_{n} \subseteq E_{n+1}$ for each $n$ and $X=\cup E_{n}$.

For each $n, D_{n}$, viewed as an operator in $\mathcal{Q}$, is the multiplication by some real-valued, bounded, measurable function $g_{n}$ satisfying $g_{n} \chi_{n}=g_{n}$ where $\chi_{n}$ is the characteristic function of $E_{n}$.

We can now define a measurable function $g$ on $X$ by $g \chi_{n}=g_{n}$. Since $D_{m} P_{n}=D_{n}$, $g_{m} \chi_{n}=g_{n}$ for $m>n$; and $g$ is well defined.

Let $\mathscr{D}(D)$ be the dense linear subspace $\{f \in H: g f \in H\}$ and $D$ be the (not necessarily bounded) linear operator of multiplication by the function $g$, defined on $\mathcal{D}(D)$. The operator $D$, such defined, is affiliated with the algebra $\mathcal{Q}$.

For each $f$ in $H, P_{n} f \in \mathscr{D}(D)$ and $D P_{n} f=D_{n} f=D_{n} P_{n} f$ because $g \chi_{n} f=g_{n} f$. Since $\delta_{P_{n}}=\operatorname{ad}\left(D_{n}\right)$,

$$
\delta\left(P_{n} U P_{n}\right) f=D_{n} P_{n} U P_{n} f-P_{n} U P_{n} D_{n} f=D P_{n} U P_{n} f-P_{n} U D P_{n} f .
$$

Thus if $f \in \mathscr{Q}(D) \cap U^{*} \mathscr{D}(D)$ then

$$
\delta\left(P_{n} U P_{n}\right) f=C(U) P_{n} U P_{n} f=(D U-U D) U^{*} P_{n} U P_{n} f
$$


and, since $P_{n} \uparrow I, P_{n} U^{*} P_{n} U \uparrow I$. Thus

$$
\delta(U) f=C(U) U f=D U f-U D f .
$$

Each $T$ in $\mathcal{S}+\mathcal{S}^{*}$ can be written as $\Sigma A_{U} U$, where $U \in \bar{G}$ and $A_{U} \in \mathbb{Q}$. Hence, if $U f$ is in $\mathscr{D}(D)$ for every $U$ in $\bar{G}$, then

$$
\delta(T) f=\sum \delta\left(A_{U} U\right) f=\sum A_{U} \delta(U) f=\sum A_{U}(D U f-U D f) .
$$

Since $A D f=D A f$ for each $f \in \mathscr{D}(D)$ and $A \in \mathcal{Q}$,

$$
\delta(T) f=\sum\left(D A_{U} U f-A_{U} D f\right)=D T f-T D f .
$$

We now discuss the existence of a bounding sequence for a given derivation. We will need the following lemma.

LEMMA 4.4 Let $\delta$ be a derivation in $D\left(\delta+\delta^{*}, \mathscr{Q}\right)$ and $E_{0}, E_{1}, \ldots, E_{n}$ be projections in $Q$ such that:

(1) For each $j$ there is $U_{j}$ in $\bar{G}$ such that $U_{j}^{*} E_{j} U_{j} \leqslant E_{0}$.

(2) $E_{1}, E_{2}, \ldots, E_{n}$ are pairwise orthogonal.

(3) The restriction of $\delta$ to $E_{0}\left(\delta+\varsigma^{*}\right) E_{0}$ is bounded.

Then the restriction of $\delta$ to $F\left(\delta+\delta^{*}\right) F$ is bounded, where $F=E_{1}+E_{2}+\cdots+E_{n}$.

Proof. Let $K$ be the norm of the restriction of $\delta$ to $E_{0}\left(\delta+\delta^{*}\right) E_{0}$ and let $F_{j}$, for $j \geqslant 1$, be $U_{j}^{*} E_{j} U_{j}$. For $T$ in $F\left(\delta+\varsigma^{*}\right) F$,

$$
T=\sum_{j, k \geqslant 1} E_{j} T E_{k}=\sum_{j, k \geqslant 1} U_{j} F_{j} U_{j}^{*} T U_{k} F_{k} U_{k}^{*}=\sum_{j, k \geqslant 1} U_{j} T_{j k} U_{k}^{*}
$$

where $T_{j k} \in E_{0}\left(\delta+\varsigma^{*}\right) E_{0}$ and $\left\|T_{j k}\right\| \leqslant\|T\|$. Hence,

$$
\begin{aligned}
\delta(T) & =\sum_{j, k \geqslant 1}\left(\delta\left(U_{j}\right) T_{j k} U_{k}^{*}+U_{j} \delta\left(T_{j k}\right) U_{k}^{*}+U_{j} T_{j k} \delta\left(U_{k}^{*}\right)\right) \\
& \leqslant \sum_{j, k \geqslant 1}\left(\left\|\delta\left(U_{j}\right)\right\|\left\|T_{j k}\right\|+\left\|\delta\left(T_{j k}\right)\right\|+\left\|T_{j k}\right\|\left\|\delta\left(U_{k}^{*}\right)\right\|\right) \\
& \leqslant\|T\| \sum_{j, k \geqslant 1}\left(\left\|\delta\left(U_{j}\right)\right\|+K+\left\|\delta\left(U_{k}^{*}\right)\right\|\right)=\|T\| M
\end{aligned}
$$

where $M$ is a real number independent of $T$.

LEMMA 4.5. Let $E_{0}$ be a nonzero projection in $Q$. Then there is a sequence $\left\{E_{i}\right\}$ of pairwise orthogonal projections in $\mathbb{Q}$ with sum I such that for each i there is some $U_{i}$ in $G$ such that $U_{i}^{*} E_{i} U_{i} \leqslant E_{0}$.

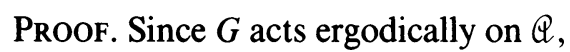

$$
I=\bigvee\left\{U E_{0} U^{*}: U \in G\right\}
$$

Let $\left\{E_{i}\right\}$ be a maximal set of pairwise orthogonal projections in $\mathbb{Q}$ such that for each $i$ there is some $U_{i}$ in $G$ such that $U_{i}^{*} E_{i} U_{i} \leqslant E_{0}$. The existence of such a set is guaranteed by the Zorn's Lemma (the set is countable since $H$ is separable).

If $I-\Sigma E_{i}$ (denoted $E$ ) is a nonzero projection, then there is some $U$ in $G$ such that $U E_{0} U^{*} E \neq 0$. We can, therefore, add $U E_{0} U^{*} E$ to $\left\{E_{i}\right\}$ and, since $U^{*}\left(U E_{0} U^{*} E\right) U \leqslant E_{0}$, it will contradict the maximality of $\left\{E_{i}\right\}$. Thus $\Sigma E_{i}=I$. 
Corollary 4.6. Let $\delta$ be a derivation in $D\left(\delta+\delta^{*}, \mathcal{Q}\right)$. If there is a nonzero projection $F_{0}$ in $Q$ such that the restriction of $\delta$ to $E_{0}\left(\delta+\mathcal{S}^{*}\right) E_{0}$ is bounded, then $\delta$ has a bounding sequence.

Proof. Let $\left\{E_{i}\right\}$ be the set given by the previous lemma and let $P_{n}$ be $\Sigma_{1 \leqslant n} E_{i}$. By Lemma 4.4, the restriction of $\delta$ to $P_{n}\left(\delta+\delta^{*}\right) P_{n}$ is bounded and, since $\Sigma E_{i}=I$, $P_{n} \uparrow 1$.

We conclude:

THEOREM 4.7. Let $\delta$ be a derivation in $D\left(\mathcal{S}+\mathcal{S}^{*}, \mathfrak{Q}\right)$. Then the following are equivalent:

(1) There is a nonzero projection $E$ in $Q$ such that

$$
\operatorname{Sup}\{\|\delta(E U E)\|: U \in \bar{G}\}<\infty .
$$

(2) There is a selfadjoint linear operator $D$, affiliated with $Q$, such that for each $T$ ( $\left.=A_{0}+A_{1} U_{1}+\cdots+A_{n} U_{n}\right)$ in $\mathfrak{S}+\mathcal{S}^{*}$ and each $f$ in a dense subspace of $H$, namely $\left\{f \in H: f \in \mathscr{Q}(D), U_{k} f \in \mathscr{D}(D)\right.$ for each $\left.1 \leqslant k \leqslant n\right\}$, we have $\delta(T) f=D T f-T D f$.

Proof. (1) implies (2): Theorem 4.3, combined with Corollary 4.6, proves that, for each $f$ in $\left\{f \in H: f \in \mathscr{D}(D), U_{k} f \in \mathscr{D}(D)\right.$ for each $\left.1 \leqslant k \leqslant n\right\}$, we have $\delta\left(U_{k}\right) f=$ $D U_{k} f-U_{k} D f$ for each $k \leqslant n$. Therefore,

$$
\delta(T) f=A_{1} \delta\left(U_{1}\right) f+\cdots+A_{n} \delta\left(U_{n}\right) f=D T f-T D f .
$$

It is left to prove that the subspace is dense in $H$.

As $D$ is affiliated with the maximal abelian algebra of multiplications by functions in $L^{\infty}(X, m), D$ is the operator of multiplication by some measurable function $g$ (and $D$ is defined on $\mathscr{D}(D)=\{f \in H: g f \in H\}$ which is dense in $H$ ) and, thus, there is a sequence of projections $\left\{E_{j}\right\}$ in $\mathbb{Q}$ such that $E_{j} \uparrow I$ and, for each $j, D E_{j} \in \mathbb{Q}$. Let $F_{j}$ be the projection $E_{j} U_{1}^{*} E_{j} U_{1} U_{2}^{*} E_{j} U_{2} \cdots U_{n}^{*} E_{j} U_{n}$. Then $F_{j} \uparrow I$ and, for each $j$ and each $f$ in $F_{j}(H), f$ is in $\mathscr{D}(D)$ and so is $U_{k} f$ for every $1 \leqslant k \leqslant n$. This completes the proof that the subspace is dense in $H$.

(2) implies (1): Let $U$ be in $\bar{G}$ and $E$ be any nonzero projection in $Q$ such that $D E$ is in $Q$. For every function $h$ in $U^{*} E U E(H), h$ and $U h$ are in $\mathscr{D}(D)$. Hence, for every $f$ in $H$,

$$
\delta(E U E) f=\delta(U) U^{*} E U E f=D E U E f-U D U^{*} E U E f=D E U E f-U D E U^{*} E U f .
$$

Therefore, $\|\delta(E U E)\| \leqslant 2\|D E\|$ and, since the right-hand side is independent of $U$, $\operatorname{Sup}\{\|\delta(E U E)\|: U \in \bar{G}\}<\infty$.

EXAMPLe 4.8. Let $X$ be $\mathbf{R}$ and $m$ be Lebesgue measure on R. Fix a negative irreducible number $u$ and define the set $S$ in $\mathbf{R}: S=\{a u-r: r \geqslant 0, r \in \mathbf{Q}, a>0$, $a \in \mathbf{Q}\} \cup\{b u+r: r, b \in \mathbf{Q}, b \geqslant 0, r \geqslant 0\}$. Let $G$ be the semigroup of translations by $s$ in $S$, i.e. $U$ in $G$ (to be denoted $U_{s}$ ) is of the form $U f(t)=f(t-s$ ) for some $s$ in $S$, where $f$ is in $L^{2}(X, m)$. It can be seen [8, Example 1.9] that $G$ is an ordered semigroup that acts freely and ergodically on $Q$ (the multiplication algebra on $\left.L^{2}(X, m)\right)$. Let $\mathcal{S}$ be the algebra generated by $\mathcal{Q}$ and $G$, then $\mathcal{S}$ is an irreducible triangular algebra.

Let $\delta$ be defined by $\delta\left(A U_{s}\right)=s A U_{s}, A \in \mathbb{Q}, U_{s} \in \bar{G}$, and by linearity. Then $\delta$ is in $D\left(\mathcal{S}+\varsigma^{*}, \mathcal{Q}\right)$. Let $E$ be the projection in $\mathscr{Q}$ which is the multiplication by the 
characteristic function of the interval $(0,1)$. For $s$ in $S, E U_{s} E \neq 0$ only if $|s|<1$, hence

$$
\left\|\delta\left(E U_{s} E\right)\right\|=\left\|s E U_{s} E\right\| \leqslant 1, \quad s \in S .
$$

Therefore, there is a linear operator $D$, as in Theorem 4.7, satisfying: For each $T$ in $\delta+\delta^{*}, \delta(T)=D T-T D$ on a dense supspace of $H$. In fact, this operator is just the operator of multiplication by the function $g(t)=t$ and is defined on $\{f \in H$ : $g f \in H\}$.

EXAmple 4.9. Let $X$ be any locally compact Hausdorff space with a $\sigma$-finite regular Borel measure. The Hilbert space $H$ would be $L^{2}(X, m)$ and $Q$ will be the algebra of multiplication by functions in $L^{\infty}(X, m)$. Let $U$ be a unitary operator acting ergodically on $\mathcal{Q}$ and assume that $X$ is an infinite set. Then, by [8, Lemma 1.7] the algebra $\delta$ (generated by $Q$ and $U$ ) is triangular irreducible.

Let $\delta$ be defined by: $\delta\left(A U^{k}\right)=k A U^{k}, A \in \mathbb{Q}, k \in \mathbf{Z}$, and by linearity. Then $\delta$ is in $D\left(\delta+\varsigma^{*}, \mathcal{Q}\right)$. Let $E$ be any projection, different from 0 , in $\mathcal{Q}$. By ergodicity, we can find a sequence $k(n)$ of integers such that, for each $n, E U^{k(n)} E U^{-k(n)} \neq 0$; hence $\left\|\delta\left(E U^{k(n)} E\right)\right\|=k(n)$. Therefore there is no operator that implements $\delta$ in the sense of Theorem 4.7 .

\section{REFERENCES}

1. W. B. Arveson, On groups of automorphisms of operator algebras, J. Funct. Anal. 15 (1974), 217-243.

2. N. Dunford and J. T. Schwartz, Linear operators, Part I: General theory, Interscience, New York, 1958.

3. F. P. Greenleaf, Invariant means on topological groups, Van Nostrand Math. Studies, No. 16, Van Nostrand-Rheinhold, New York, 1969.

4. R. V. Kadison and I. M. Singer, Triangular operator algebras, Amer. J. Math. 82 (1960), 227-259.

5. G. K. Pederson, $C^{*}$-algebras and their automorphism groups, Academic Press, London, 1979.

6. J. R. Ringrose, Cohomology of operator algebras, Lectures on Operator Algebras, Springer-Verlag, Berlin and New York, 1972.

7. S. Sakai, $C^{*}$-algebras and $W^{*}$-algebras, Ergebnisse der Math. und ihrer Grenzgebiete, no. 60, Springer-Verlag, Berlin and New York, 1971.

8. B. Solel, Irreducible triangular algebras, Thesis, Univ. of Pennsylvania, 1981.

Department of Mathematics, Tel-Aviv University, Tel-Aviv, Israel 